\title{
Synthetic Studies Toward FR182877. Remarkable Solvent Effect in the Vinylogous Morita-Baylis-Hillman Cyclization
}

\author{
Joey L. Methot and William R. Roush* \\ Department of Chemistry, University of Michigan, Ann Arbor, MI, 48109 \\ email:roush@umich.edu
}

SUPPORTING INFORMATION 
General Methods. Reactions were performed in dry glassware under an atmosphere of nitrogen. Tetrahydrofuran, dichloromethane and diethyl ether were purified by passage over activated A-1 alumina. ${ }^{1}$ All other reagents and solvents were used as received from commercial sources. Analytical thin layer chromatography (TLC) was performed on Kieselgel $60 \mathrm{~F}_{254}$ glass plates precoated with a $0.25 \mathrm{~mm}$ thickness of silica gel. The TLC plates were visualized with UV light and/or by staining with ceric ammonium molybdate or potassium permanganate. Flash column chromatography was performed according to the method of Still ${ }^{2}$ using Kieselgel 60 (230-400 mesh) silica gel or $\mathrm{pH}=7.7$ neutral silica gel. Proton and carbon-13 nuclear magnetic resonance spectra $\left({ }^{1} \mathrm{H}\right.$ and ${ }^{13} \mathrm{C}$ NMR) were recorded in $\mathrm{CDCl}_{3}$ on a Varian VXR-400 spectrometer or on a Varian Inova-500 spectrometer. The proton signal of residual, nondeuterated $\mathrm{CHCl}_{3}(\square 7.27 \mathrm{ppm})$ and the carbon-13 signal of $\mathrm{CDCl}_{3}(\square 77.2 \mathrm{ppm})$ were used as internal references. Coupling constants are reported in Hz. Infrared (IR) spectra were recorded as thin films on a Perkin-Elmer Spectrum 1000 FTIR. Optical rotations were measured on a Rudolph Autopol III polarimeter using a quartz cell with $1 \mathrm{~mL}$ capacity and a $10 \mathrm{~cm}$ path length. Mass spectra were recorded on a VG 70-250-S spectrometer manufactured by Micromass Corp. (Manchester UK). HPLC purifications were performed using a HPLC system composed of two Rainin HPXL pumps connected to either various Dynamax ${ }^{\circledR}$ axial compression columns packed with Rainin $60 \AA$ irregular silica gel. Samples were loaded into the system with a $2 \mathrm{~mL}$ Rheodyne 7125 injector and were detected using a either Rainin Dynamax ${ }^{\circledR}$ UV-C detector or a Rainin Dynamax ${ }^{\circledR}$ RI-1 detector. Integration of the various signals was performed using the reprocessing program within the Dynamax ${ }^{\circledR}$ HPLC Method Manager.

\footnotetext{
${ }^{1}$ Pangborn, A. B.; Giardello, M. A.; Grubbs, R. H.; Rosen, R. K.; Timmers, F. J. Organometallics 1996, $15,1518$.

${ }^{2}$ Still, W. C.; Kahn, M.; Mitra, A. J. Org. Chem. 1978, 43, 2923.
} 

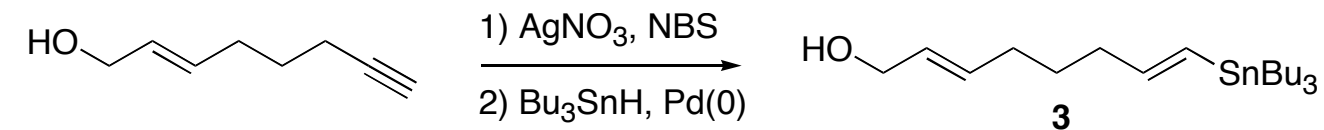

$(\boldsymbol{E}, \boldsymbol{E})$-8-(Tributylstannanyl)-octa-2,7-dien-1-ol (3). A suspension of (E)-2-octen-7-yn-1-ol ${ }^{3}$ (400 mg, $3.22 \mathrm{mmol})$ and $N$-bromosuccinimide $(600 \mathrm{mg}, 3.37 \mathrm{mmol})$ in acetone $(10 \mathrm{~mL})$ was treated with $\mathrm{AgNO}_{3}(50 \mathrm{mg}, 0.29 \mathrm{mmol})$ and stirred for $1 \mathrm{~h} .{ }^{4}$ The mixture was diluted with ether and washed with $10 \% \mathrm{HCl}, 10 \% \mathrm{KOH}$, dried $\left(\mathrm{Na}_{2} \mathrm{SO}_{4}\right)$, filtered and concentrated. The oily residue was dissolved in THF $(10 \mathrm{~mL})$ and treated with $\mathrm{Pd}\left(\mathrm{PPh}_{3}\right)_{4}(40 \mathrm{mg}, 0.035 \mathrm{mmol})$ and cooled to $0{ }^{\circ} \mathrm{C}$. $\mathrm{Bu}_{3} \mathrm{SnH}(1.90 \mathrm{~mL}, 7.06 \mathrm{mmol})$ was added dropwise over $15 \mathrm{~min}$, the mixture was stirred for $1 \mathrm{~h}$ and finally concentrated to an oil. Purification of the crude product by chromatography on neutral $\mathrm{SiO}_{2}$ (ether/hexanes, $0: 1$ to $1: 1$ ) gave $1.03 \mathrm{~g} \mathrm{(77 \% )}$ of the desired vinyl stannane 3: $R_{f} 0.28$ (ether/hexanes, $\left.1: 1\right) ;{ }^{1} \mathrm{H}$ NMR (500 MHz) $\square 6.00-5.80(\mathrm{~m}, 2 \mathrm{H}), 5.74-$ $5.62(\mathrm{~m}, 2 \mathrm{H}), 4.11(\mathrm{t}, 2 \mathrm{H}, J=5.6 \mathrm{~Hz}), 2.16(\mathrm{dt}, 2 \mathrm{H}, J=7.3,5.6 \mathrm{~Hz}), 2.07$ (q, $2 \mathrm{H}, J=7.6 \mathrm{~Hz})$, 1.53-1.47 (m, 8 H), 1.35-1.28 (m, 6 H), 1.22 (t, 1 H, $J=5.9$ Hz), 0.94-0.86 (m, $6 \mathrm{H}), 0.90(\mathrm{t}, 9 \mathrm{H}$, $J=7.3 \mathrm{~Hz}) ;{ }^{13} \mathrm{C}$ NMR $(100 \mathrm{MHz}) \square 149.3,133.3,129.3,127.8,63.9,37.4,31.8,29.3,28.5,27.4$, 13.9, 9.6; IR (neat) 3326, 2956, 2926, 2872, 2854, 1599, $1464 \mathrm{~cm}^{-1}$; HRMS (EI), calcd for $\mathrm{C}_{20} \mathrm{H}_{40} \mathrm{OSnNa} 439.1999[\mathrm{M}+\mathrm{Na}]^{+}$, found $439.2003 \mathrm{~m} / \mathrm{z}$.
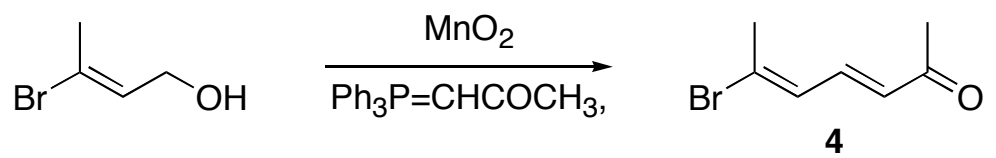

(E,E)-6-Bromohepta-3,5-dien-2-one (4). A suspension of (E)-3-bromo-2-buten-1-ol ${ }^{5}$ (245 $\mathrm{mg}, 1.62 \mathrm{mmol}), \mathrm{MnO}_{2}(1.41 \mathrm{~g}, 16.2 \mathrm{mmol})$ and 1-triphenylphosporanylidene-2-propanone (1.00 g, $3.14 \mathrm{mmol})$ in $\mathrm{CH}_{2} \mathrm{Cl}_{2}(5 \mathrm{~mL})$ was stirred vigorously for $15 \mathrm{~h} .{ }^{6}$ The mixture was filtered through Celite, concentrated and chromatographed on $\mathrm{SiO}_{2}\left(\mathrm{CH}_{2} \mathrm{Cl}_{2}\right)$ giving $207 \mathrm{mg}(68 \%)$ of the dienyl ketone 4: $R_{f} 0.50$ (ether/hexanes, $\left.1: 1\right) ;{ }^{1} \mathrm{H}$ NMR $(500 \mathrm{MHz}) \square 7.20(\mathrm{dd}, 1 \mathrm{H}, J=15.4$, $11.7 \mathrm{~Hz}), 6.62(\mathrm{dq}, 1 \mathrm{H}, J=11.5,1.2,1.2,1.2 \mathrm{~Hz}), 6.17(\mathrm{~d}, 1 \mathrm{H}, J=15.1 \mathrm{~Hz}), 2.50(\mathrm{~d}, 3 \mathrm{H}, J=$ $1.0 \mathrm{~Hz}), 2.29$ (s, $3 \mathrm{H}) ;{ }^{13} \mathrm{C}$ NMR (100 MHz) $\square$ 197.9, 136.1, 133.0, 130.6, 129.8, 28.0, 24.4; IR

\footnotetext{
${ }^{3}$ Harvey, D. F.; Lund, K. P.; Neil, D. A. J. Am. Chem. Soc. 1992, 114, 8424.

${ }^{4}$ Boden, C. D. J., Pattenden, G.; Ye, T. J. Chem. Soc., Perkin Trans. 1 1996, 2417.

${ }^{5}$ Roush, W. R.; Brown, B. B. J. Am. Chem. Soc. 1993, 115, 2268.
} 
(neat) $1661,1640,1620 \mathrm{~cm}^{-1}$; HRMS (EI), calcd for $\mathrm{C}_{7} \mathrm{H}_{9} \mathrm{OBr} 187.9837[\mathrm{M}]^{+}$, found 187.9840 $\mathrm{m} / \mathrm{z}$.

Stereochemical assignment for $\mathbf{4}$ is based on ${ }^{1} \mathrm{H}$ NMR coupling contants and NOE data:
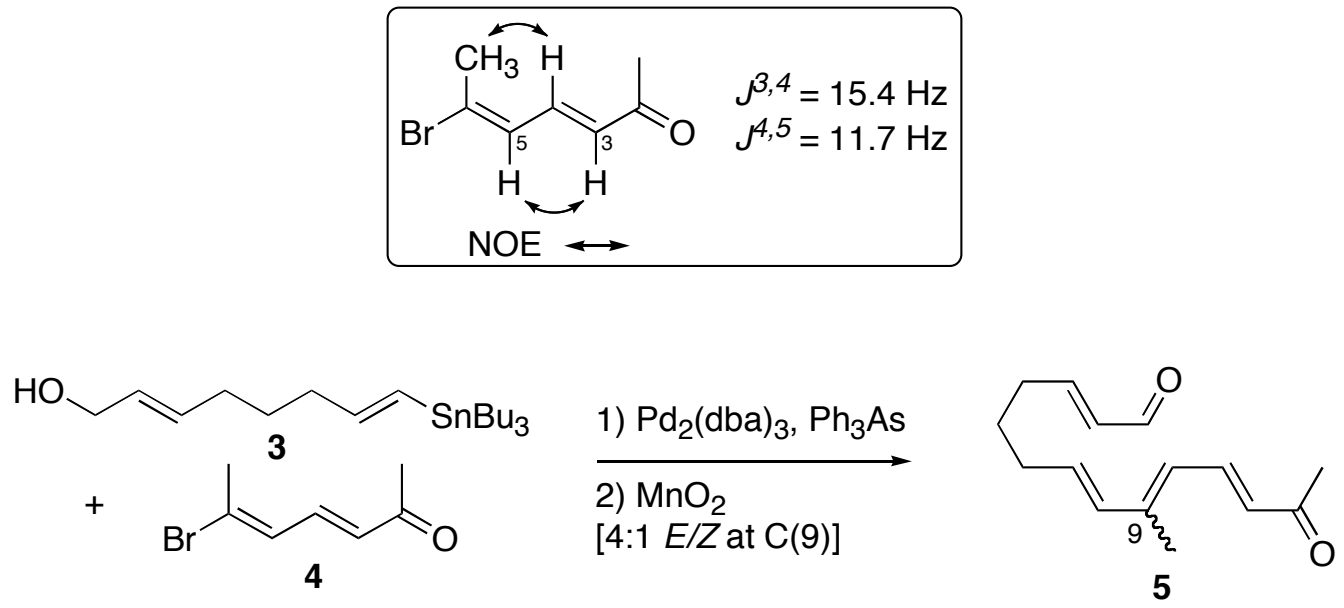

[2(E),7(E),9(E),11(E)]-9-Methyl-13-oxo-tetradeca-2,7,9,11-tetraenal (5). A solution of the vinyl stannane 3 (1.03 g, $2.48 \mathrm{mmol}$ ) and the vinyl bromide 4 (300 $\mathrm{mg}, 1.59 \mathrm{mmol}$ ) in THF $(10 \mathrm{~mL})$ was treated with $\mathrm{Pd}_{2}(\mathrm{dba})_{3} \cdot \mathrm{CHCl}_{3}(80 \mathrm{mg}, 0.077 \mathrm{mmol})$ and $\mathrm{Ph}_{3} \mathrm{As}(95 \mathrm{mg}, 0.31 \mathrm{mmol})$. The mixture was stirred for $13 \mathrm{~h}$ and concentrated. Separation of the mixture by chromatography on neutral $\mathrm{SiO}_{2}$ (ether/hexanes, 1:4 to 3:2) gave $305 \mathrm{mg}(82 \%)$ of the tetraenyl ketone as an inseparable 4:1 E/Z olefin mixture at $\mathrm{C}(9){ }^{7}$ The oil was dissolved in $\mathrm{CH}_{2} \mathrm{Cl}_{2}(20 \mathrm{~mL})$, treated with $\mathrm{MnO}_{2}(1.00 \mathrm{~g}, 11.5 \mathrm{mmol})$, stirred for $3 \mathrm{~h}$ and treated with additional $\mathrm{MnO}_{2}(300 \mathrm{mg}, 3.45$ mmol). The suspension was stirred another $2 \mathrm{~h}$, filtered through a pad of Celite and then through a pad of neutral $\mathrm{SiO}_{2}$ (washing with ether). Concentration of the filtrate provided $278 \mathrm{mg}(92 \%)$ of the desired aldehyde 5 as an inseparable 4:1 E/Z olefin mixture at $\mathrm{C}(9):{ }^{1} \mathrm{H}$ NMR $(500 \mathrm{MHz})$ $\square 9.53(\mathrm{~d}, 1 \mathrm{H}, J=7.8 \mathrm{~Hz}), 7.54(\mathrm{dd}, 1 \mathrm{H}, J=15.1,11.7 \mathrm{~Hz}), 6.86(\mathrm{dt}, 1 \mathrm{H}, J=15.6,6.8,6.8 \mathrm{~Hz})$, 6.12-6.22 (m, $4 \mathrm{H}), 5.94$ (dt, $1 \mathrm{H}, J=15.6,7.1,7.1 \mathrm{~Hz}), 2.39$ (q, $2 \mathrm{H}, J=7.4 \mathrm{~Hz}), 2.31$ (s, $3 \mathrm{H})$, $2.25(\mathrm{q}, 2 \mathrm{H}, J=7.1 \mathrm{~Hz}), 2.01(\mathrm{~d}, 3 \mathrm{H}, J=1.0 \mathrm{~Hz}), 1.68(\mathrm{dq}, 2 \mathrm{H}, J=7.6,7.6,7.6,7.6 \mathrm{~Hz}) ;{ }^{13} \mathrm{C}$ NMR (125 MHz) $\square$ 199.0, 198.8, 145.4, 139.6, 134.7, 134.6, 132.7, 129.7, 129.6, 127.4, 32.8,

\footnotetext{
${ }^{6}$ Wei, X.; Taylor, R. J. K. J. Org. Chem. 2000, 65, 616.

${ }^{7}$ The $E / Z$ mixture is at the trisubstituted olefin. Isomerization was also observed in the presence of LiCl, PPTs and $\mathrm{SiO}_{2}$. For related examples see (a) Laurent, A.; Prat, V.; Valla, A.; Andriamialisoa, Z.; Giraud, M.; Labia, R.;

Potier, P. Tetrahedron Lett. 2000, 41, 7221; (b) Andriamialisoa, Z.; Valla, A.; Zennache, S.; Giraud, M.; Potier, P. Tetrahedron Lett. 1993, 34, 8091; (c) Lugtenburg, J. Pure \& Appl. Chem. 1985, 57, 753; (d) Liu, R. S. H.; Asato, A. E.; Denny, M. J. Am. Chem. Soc. 1977, 99, 8095; (e) Stork, G.; Kraus, G. A. J. Am. Chem. Soc. 1976, 98, 2351.
} 
31.9, 31.8, 28.8, 27.9; IR (neat) 2927, 1688, 1652, 1590, $1572 \mathrm{~cm}^{-1}$; HRMS (EI), calcd for $\mathrm{C}_{15} \mathrm{H}_{20} \mathrm{O}_{2} 232.1463[\mathrm{M}]^{+}$, found $232.1458 \mathrm{~m} / \mathrm{z}$.

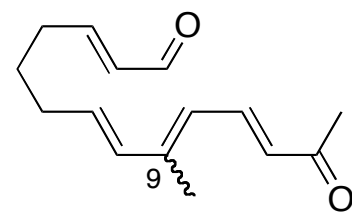

$5[4: 1 E / Z$ at $C(9)]$

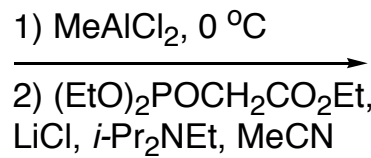

$\mathrm{LiCl}, i-\mathrm{Pr}_{2} \mathrm{NEt}, \mathrm{MeCN}$

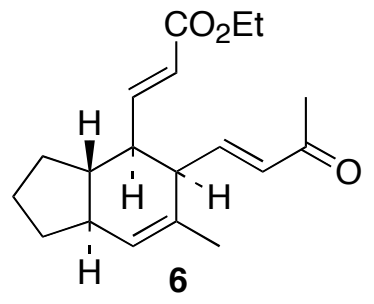

rac-[3a $(S), 4(S), 5(S), 7 \mathrm{a}(S)]-3-[6-M e t h y l-5-(3-o x o-b u t-1(E)$-enyl)-2,3,3a,4,5,7a-hexahydro$1 \boldsymbol{H}$-inden-4-yl]-(E)-acrylic Acid Ethyl Ester (6). A solution of aldehyde 5 as a 4:1 E/Z olefin mixture at $\mathrm{C}(9)(70 \mathrm{mg}, 0.30 \mathrm{mmol})$ in $\mathrm{CH}_{2} \mathrm{Cl}_{2}(3 \mathrm{~mL})$ was treated at $-78{ }^{\circ} \mathrm{C}$ with a $1 \mathrm{M}$ solution of $\mathrm{MeAlCl}_{2}$ in hexane $(0.25 \mathrm{~mL}, 0.25 \mathrm{mmol})$ and warmed to $0{ }^{\circ} \mathrm{C}$. The mixture was stirred for 1 h, quenched with $\mathrm{pH}=7$ buffer solution and partitioned between $\mathrm{CH}_{2} \mathrm{Cl}_{2}$ and water. The organic phase was dried $\left(\mathrm{Na}_{2} \mathrm{SO}_{4}\right)$, filtered and concentrated. Purification of the crude product by chromatography on neutral $\mathrm{SiO}_{2}$ (ether/hexanes, 1:9 to 3:7) gave $14 \mathrm{mg}$ (20\%) of recovered unsaturated aldehyde 5 (1:4 E/Z from crude ${ }^{1} \mathrm{H}$ NMR analysis) and $36 \mathrm{mg}(52 \%)$ of the DielsAlder product (>15:1 diastereomeric purity): $R_{f} 0.38$ (ether/hexanes, $\left.1: 1\right) ;{ }^{1} \mathrm{H}$ NMR $(500 \mathrm{MHz}) \square$ $9.68(\mathrm{~d}, 1 \mathrm{H}, J=2.4 \mathrm{~Hz}), 6.66(\mathrm{dd}, 1 \mathrm{H}, J=16.1,9.5 \mathrm{~Hz}), 6.09$ (d, $1 \mathrm{H}, J=16.1 \mathrm{~Hz}), 5.79(\mathrm{br} \mathrm{s}, 1$ H), $3.30(\mathrm{dd}, 1 \mathrm{H}, J=8.8,5.9 \mathrm{~Hz}), 2.68(\mathrm{ddd}, 1 \mathrm{H}, J=11.5,5.6,2.2 \mathrm{~Hz}), 2.25(\mathrm{~s}, 3 \mathrm{H}), 2.03(\mathrm{dq}$, $1 \mathrm{H}, J=11.7,6.1,6.1,6.1 \mathrm{~Hz}), 1.95-1.85$ (m, $2 \mathrm{H}), 1.82-1.76$ (m, $2 \mathrm{H}), 1.69$ (dtd, $1 \mathrm{H}, J=11.5$, 11.5, 11.5, $6.3 \mathrm{~Hz}), 1.62$ (s, $3 \mathrm{H}), 1.25-1.17(\mathrm{~m}, 2 \mathrm{H}) ;{ }^{13} \mathrm{C}$ NMR $(125 \mathrm{MHz}) \square 203.2,198.1$, 145.5, 133.4, 131.9, 127.9, 56.3, 46.1, 45.2, 39.4, 28.5, 27.2, 27.1, 22.3, 21.6; IR (neat) 2953, 2871, 1721, 1673, $1619 \mathrm{~cm}^{-1}$; HRMS (EI), calcd for $\mathrm{C}_{15} \mathrm{H}_{20} \mathrm{O}_{2} 232.1463[\mathrm{M}]^{+}$, found 232.1465 $\mathrm{m} / \mathrm{z}$.

A suspension of the cycloadduct ( $65 \mathrm{mg}, 0.28 \mathrm{mmol}), \mathrm{LiCl}(20 \mathrm{mg}, 0.48 \mathrm{mmol})$ and $(\mathrm{EtO})_{2} \mathrm{P}(\mathrm{O}) \mathrm{CH}_{2} \mathrm{CO}_{2} \mathrm{Et}(0.10 \mathrm{~mL}, 0.50 \mathrm{mmol})$ in $\mathrm{MeCN}(3 \mathrm{~mL})$ was treated at $-20{ }^{\circ} \mathrm{C}$ with $i$ $\mathrm{Pr}_{2} \mathrm{NEt}(0.080 \mathrm{~mL}, 0.45 \mathrm{mmol})$ and stirred for $1 \mathrm{~h}^{8}{ }^{8}$ The mixture was slowly warmed to $23{ }^{\circ} \mathrm{C}$ over $5 \mathrm{~h}$, stirred another $10 \mathrm{~h}$, poured into ether, washed with water, dried $\left(\mathrm{Na}_{2} \mathrm{SO}_{4}\right)$, filtered and concentrated. Chromatography of the crude product on $\mathrm{SiO}_{2}$ (ether/hexanes, 1:9 to 2:3) gave 61 
mg (72\%) of the ethyl ester 6: $R_{f} 0.32$ (ether/hexanes, $\left.1: 2\right) ;{ }^{1} \mathrm{H}$ NMR $(500 \mathrm{MHz}) \square 6.78(\mathrm{dd}$, $\left.\mathrm{CH}=\mathrm{CHCO}_{2} \mathrm{Et}, J=15.4,9.8 \mathrm{~Hz}\right), 6.66(\mathrm{dd}, \mathrm{CH}=\mathrm{CHCOMe}, J=15.9,9.3 \mathrm{~Hz}), 6.04(\mathrm{~d}$, $\mathrm{CH}=\mathrm{C} H \mathrm{COMe}, J=15.9 \mathrm{~Hz}), 5.85\left(\mathrm{~d}, \mathrm{CH}=\mathrm{CHCO} \mathrm{Et}_{2}, J=15.6 \mathrm{~Hz}\right), 5.77(\mathrm{~s}, \mathrm{H}-7), 4.19(\mathrm{q}$, $\mathrm{CO}_{2} \mathrm{CH}_{2} \mathrm{CH}_{3}, J=7.1 \mathrm{~Hz}$ ), $2.96(\mathrm{t}, \mathrm{H}-5, J=6.1 \mathrm{~Hz}), 2.60$ (ddd, $\left.\mathrm{H}-4, J=10.7,10.7,5.9 \mathrm{~Hz}\right), 2.28$ (s, $\left.\mathrm{CH}=\mathrm{CHCOCH}_{3}\right), 1.94-1.88(\mathrm{~m}, 1 \mathrm{H}), 1.88$ (m, H-7a), 1.75-1.68 (m, $\left.3 \mathrm{H}\right), 1.60$ (s, Me-6), 1.54 (dtd, $\mathrm{H}-3 \mathrm{a}, J=11.5,11.5,11.5,5.4 \mathrm{~Hz}$ ), 1.29 (t, $\left.\mathrm{CO}_{2} \mathrm{CH}_{2} \mathrm{CH}_{3}, J=7.1 \mathrm{~Hz}\right), 1.25-1.20$ (m, 1 $\mathrm{H}), 1.11-1.05$ (m, $1 \mathrm{H}) ;{ }^{13} \mathrm{C}$ NMR (125 MHz) $\square$ 198.2, 166.2, 149.2, 146.4, 133.5, 132.6, 127.7, 122.1, 60.3, 49.7, 47.1, 46.1, 42.6, 29.2, 27.7, 27.1, 21.9, 21.8, 14.2; IR (neat) 2959, 2871, 1719, $1675,1652,1621 \mathrm{~cm}^{-1}$; HRMS (EI), calcd for $\mathrm{C}_{19} \mathrm{H}_{26} \mathrm{O}_{3} \mathrm{Na} 325.1780[\mathrm{M}+\mathrm{Na}]^{+}$, found 325.1787 $\mathrm{m} / \mathrm{z}$.

Stereochemical assignment for $\mathbf{6}$ is based on ${ }^{1} \mathrm{H}$ NMR coupling contants and NOE data:
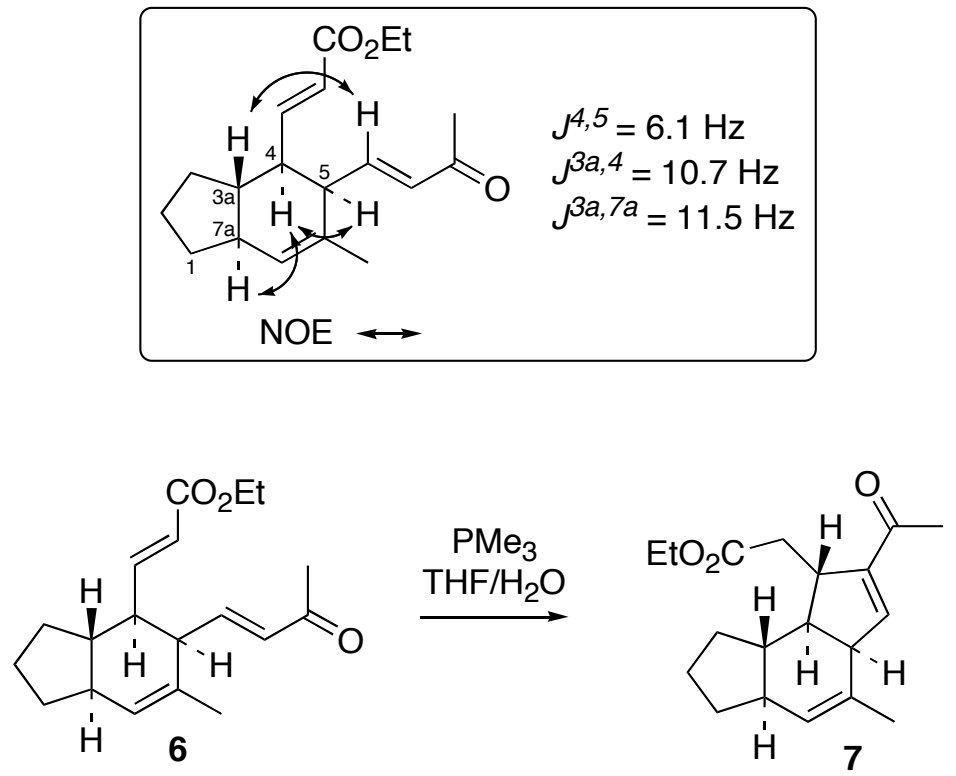

rac-[1(S),3a $(S), 5 \mathrm{a}(S), 8 \mathrm{a}(S), 8 \mathrm{~b}(S)]$-(2-Acetyl-4-methyl-1,3a,5a,6,7,8,8a,8b-octahydro-as-

indacen-1-yl)-acetic Acid Ethyl Ester (7). A solution of the enone $6(10.3 \mathrm{mg}, 0.034 \mathrm{mmol})$ in THF $(0.60 \mathrm{~mL})$ was degassed by the freeze-pump-thaw method and treated with $\mathrm{H}_{2} \mathrm{O}(0.20 \mathrm{~mL}$; also degassed) followed by $\mathrm{PMe}_{3}(7 \square \mathrm{L}, 0.065 \mathrm{mmol})$. The reaction mixture was stirred for $8 \mathrm{~h}$ until TLC analysis indicated complete substrate consumption. It was then poured into ether, washed with brine, dried $\left(\mathrm{Na}_{2} \mathrm{SO}_{4}\right)$, filtered and concentrated. ${ }^{1} \mathrm{H}$ NMR analysis of the crude

\footnotetext{
${ }^{8}$ Blanchette, M. A.; Choy, W.; Davis, J. T.; Essenfeld, A. P.; Masamune, S.; Roush, W. R.; Sakai, T. Tetrahedron Lett. 1984, 25, 2183.
} 
product indicated no significant byproduct formation. Purification of this material by chromatography on $\mathrm{SiO}_{2}$ (ether/hexanes, $1: 9$ to $3: 7$ ) gave $7.4 \mathrm{mg}(74 \%)$ of cycloadduct $7: R_{f}$ 0.46 (ether/hexanes, 1:1); ${ }^{1} \mathrm{H}$ NMR (500 MHz) $\square 6.75$ (d, H-3, $J=2.2 \mathrm{~Hz}$ ), 5.69 (br s, H-5), 4.14 and $4.13\left(2 \mathrm{q}, \mathrm{CO}_{2} \mathrm{CH}_{2} \mathrm{CH}_{3}, J=7.1 \mathrm{~Hz}\right), 3.49$ (dd, H-3a, $J=4.9,1.5 \mathrm{~Hz}$ ), 3.27 (ddd, $\mathrm{H}-1, J=$ 10.5, 3.7, $3.7 \mathrm{~Hz}$ ), 2.67 (dd, H-马, $J=15.1,3.9 \mathrm{~Hz}$ ), 2.33 (s, $\mathrm{COCH}_{3}$ ), 2.24 (dd, H-马, $J=14.9$, $10.5 \mathrm{~Hz}), 2.12$ (dd, H-8b, $J=11.5,7.1 \mathrm{~Hz}), 1.93-1.86(\mathrm{~m}, 1 \mathrm{H}), 1.87-1.76(\mathrm{~m}, 1 \mathrm{H}), 1.84(\mathrm{~d}, \mathrm{Me}-$ $4, J=0.7 \mathrm{~Hz}), 1.82(\mathrm{~m}, \mathrm{H}-5 \mathrm{a}), 1.70-1.62(\mathrm{~m}, 2 \mathrm{H}), 1.27$ (t, $\left.\mathrm{CO}_{2} \mathrm{CH}_{2} \mathrm{CH}_{3}, J=7.1 \mathrm{~Hz}\right), 1.24-1.06$ (m, 2 H), 1.10 (m, H-8a); ${ }^{13} \mathrm{C}$ NMR (125 MHz) $\square$ 197.1, 172.8, 146.0, 145.1, 132.3, 128.2, 60.6, 52.1, 49.0, 45.9, 44.9, 43.5, 37.6, 30.1, 28.5, 27.0, 22.3, 22.2, 14.6; IR (neat) 2928, 2867, 1734, $1667 \mathrm{~cm}^{-1}$; HRMS (EI), calcd for $\mathrm{C}_{19} \mathrm{H}_{26} \mathrm{O}_{3} \mathrm{Na} 325.1780[\mathrm{M}+\mathrm{Na}]^{+}$, found $325.1779 \mathrm{~m} / \mathrm{z}$.

Stereochemical assignment for $\mathbf{7}$ is based on ${ }^{1} \mathrm{H}$ NMR coupling contants and NOE data:
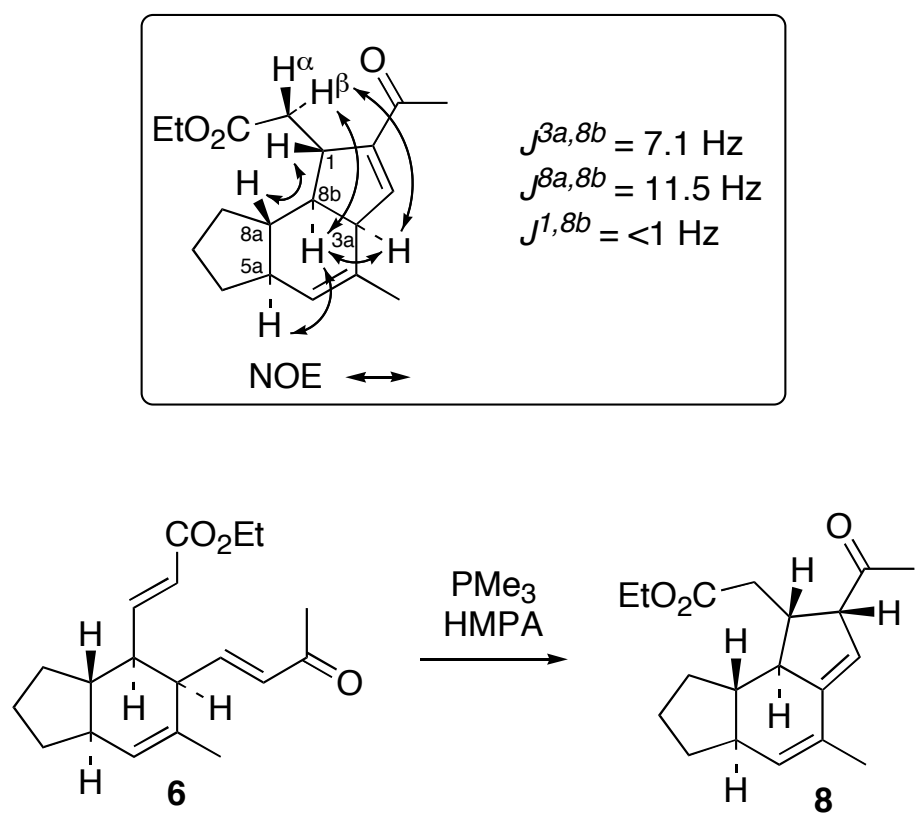

\section{rac-[1(S),2(S),5a(S),8a $(S), 8 \mathrm{~b}(S)]$-(2-Acetyl-4-methyl-1,2,5a,6,7,8,8a,8b-octahydro-as-ind-} acen-1-yl)-acetic Acid Ethyl Ester (8). A solution of the enone 6 (14.4 mg, $0.048 \mathrm{mmol}$ ) in HMPA $(1 \mathrm{~mL})$ was degassed by the freeze-pump-thaw method and treated with $\mathrm{PMe}_{3}(50 \square \mathrm{L}$, $0.46 \mathrm{mmol})$. The reaction mixture was stirred for $18 \mathrm{~h}$, poured into ether, washed with excess water, brine, dried $\left(\mathrm{Na}_{2} \mathrm{SO}_{4}\right)$, filtered and concentrated. ${ }^{1} \mathrm{H} \mathrm{NMR}$ analysis of the crude product indicated a 9:1 mixture of the olefin migration product $\mathbf{8}$ to the undesired regioisomer $\mathbf{9}$. Purification of this material by chromatography on $\mathrm{SiO}_{2}$ (ether/hexanes, $1: 9$ to 3:7) followed by 
HPLC (EtOAc/hexane, 1:4) gave $9.4 \mathrm{mg}(65 \%)$ of the olefin migration product 8: $R_{f} 0.45$ (ether/hexanes, 1:1); ${ }^{1} \mathrm{H}$ NMR (500 MHz) $\square 5.77$ (br s, H-5), 5.42 (br s, H-3), 4.10 (q, $\left.\mathrm{CO}_{2} \mathrm{CH}_{2} \mathrm{CH}_{3}, J=7.1 \mathrm{~Hz}\right), 3.65(\mathrm{br} \mathrm{d}, \mathrm{H}-2, J=8.5 \mathrm{~Hz}), 2.68(\mathrm{dd}, \mathrm{H}-\square, J=15.6,3.9 \mathrm{~Hz}), 2.67$ (dtd, H-1, $J=8.3,8.3,8.3,3.9 \mathrm{~Hz}$ ), 2.43 (dd, H-C, $J=15.6,9.8 \mathrm{~Hz}$ ), 2.36 (ddt, H-8b, $J=11.5$, 9.0, 2.4, 2.4 Hz), 2.24 (s, COCH3), 2.09-2.02 (m, 1 H), 1.93 (m, H-5a), 1.85 (dtd, 1 H, J = 12.0, 7.3, 7.3, $3.7 \mathrm{~Hz}$ ), 1.80 (t, Me-4, $J=1.7 \mathrm{~Hz}), 1.78-1.71$ (m, $2 \mathrm{H}$ ), 1.33 (dtd, H-8a, $J=11.2,11.2$, 11.2, $6.1 \mathrm{~Hz}), 1.26\left(\mathrm{t}, \mathrm{CO}_{2} \mathrm{CH}_{2} \mathrm{CH}_{3}, J=7.1 \mathrm{~Hz}\right), 1.30-1.14(\mathrm{~m}, 2 \mathrm{H}) ;{ }^{13} \mathrm{C} \mathrm{NMR}(100 \mathrm{MHz})$ 209.6, 172.8, 148.1, 131.8, 130.1, 119.2, 64.5, 60.6, 55.0, 50.5, 46.7, 44.0, 37.8, 28.7, 28.6, 28.3, 22.6, 18.8, 14.4; IR (neat) 2924, 2854, 1734, $1717 \mathrm{~cm}^{-1}$; HRMS (EI), calcd for $\mathrm{C}_{19} \mathrm{H}_{26} \mathrm{O}_{3} \mathrm{Na}$ $325.1780[\mathrm{M}+\mathrm{Na}]^{+}$, found $325.1791 \mathrm{~m} / \mathrm{z}$.

Stereochemical assignment for $\mathbf{8}$ is based on ${ }^{1} \mathrm{H}$ NMR coupling contants and NOE data:
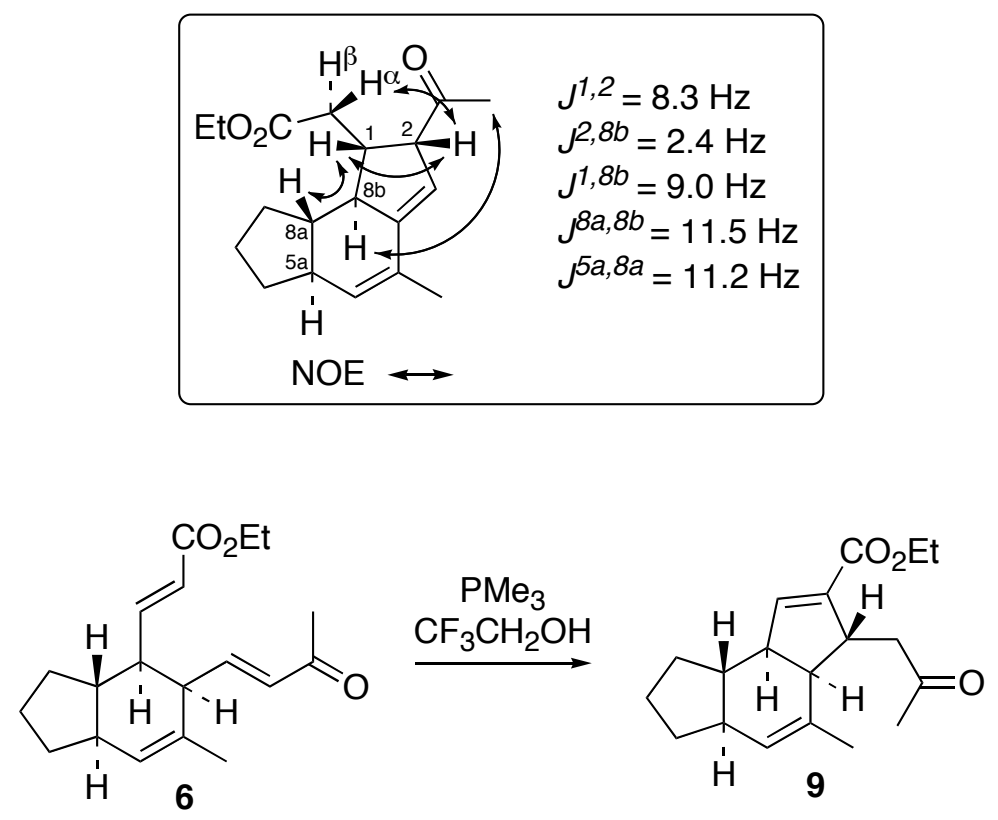

\section{rac-[3(R),3a $(S), 5 \mathrm{a}(S), 8 \mathrm{a}(S), 8 \mathrm{~b}(R)]-4-M e t h y l-3-(2-0 x 0-p r o p y l)-3,3 a, 5 a, 6,7,8,8 \mathrm{a}, 8 \mathrm{~b}-$-octa-} hydro-as-indacene-2-carboxylic Acid Ethyl Ester (9). A solution of the enone 6 (4.0 $\mathrm{mg}$, $0.013 \mathrm{mmol})$ in $\mathrm{CF}_{3} \mathrm{CH}_{2} \mathrm{OH}(0.40 \mathrm{~mL})$ was degassed by the freeze-pump-thaw method and treated with $\mathrm{PMe}_{3}(15 \square \mathrm{L}, 0.14 \mathrm{mmol})$. The reaction mixture was stirred for $1 \mathrm{~h}$ and concentrated. ${ }^{1} \mathrm{H}$ NMR analysis of the crude product indicated no significant byproduct formation. Purification of this material by chromatography on $\mathrm{SiO}_{2}$ (ether/hexanes, 1:9 to 3:7) gave $3.1 \mathrm{mg}$ (78\%) of the undesired product 9: $R_{f} 0.45$ (ether/hexanes, $\left.1: 1\right)$; ${ }^{1} \mathrm{H}$ NMR (500 MHz) 
$6.85(\mathrm{t}, \mathrm{H}-1, J=2.4 \mathrm{~Hz}), 5.63(\mathrm{br} \mathrm{s}, \mathrm{H}-4), 4.17$ and $4.15\left(2 \mathrm{q}, \mathrm{CO}_{2} \mathrm{CH}_{2} \mathrm{CH}_{3}, J=7.1 \mathrm{~Hz}\right), 3.35$ (m, H-3), 2.85 (d, $\left.\mathrm{CH}_{2} \mathrm{COCH}_{3}, J=4.9 \mathrm{~Hz}\right), 2.65$ (ddt, H-8b, J = 11.2, 5.4, 2.7, $\left.2.7 \mathrm{~Hz}\right), 2.57$ (t, H-3a, $J=8.8 \mathrm{~Hz}$ ), 2.18 (s, $\mathrm{CH}_{2} \mathrm{COCH}_{3}$ ), 1.88-1.80 (m, $\left.2 \mathrm{H}\right), 1.78$ (m, H-5a), 1.77 (br s, Me-4), 1.74-1.67 (m, $2 \mathrm{H}), 1.28\left(\mathrm{t}, \mathrm{CO}_{2} \mathrm{CH}_{2} \mathrm{CH}_{3}, J=7.1 \mathrm{~Hz}\right), 1.24-1.08(\mathrm{~m}, 2 \mathrm{H}), 0.97$ (dtd, $\mathrm{H}-8 \mathrm{a}, J=$ 10.5, 10.5, 10.5, 6.3 Hz); ${ }^{13} \mathrm{C}$ NMR (125 MHz) 207.7, 165.6, 146.8, 136.5, 135.6, 128.7, 127.2, 60.4, 50.2, 50.0, 49.0, 48.0, 44.3, 30.9, 29.6, 28.6, 23.1, 22.8, 14.5; IR (neat) 2962, 2929, 2881, $1706 \mathrm{~cm}^{-1}$; HRMS (EI), calcd for $\mathrm{C}_{19} \mathrm{H}_{27} \mathrm{O}_{3} 303.1960[\mathrm{M}+\mathrm{H}]^{+}$, found $303.1968 \mathrm{~m} / z$.

Stereochemical assignment for 9 is based on ${ }^{1} \mathrm{H}$ NMR coupling contants and NOE data:
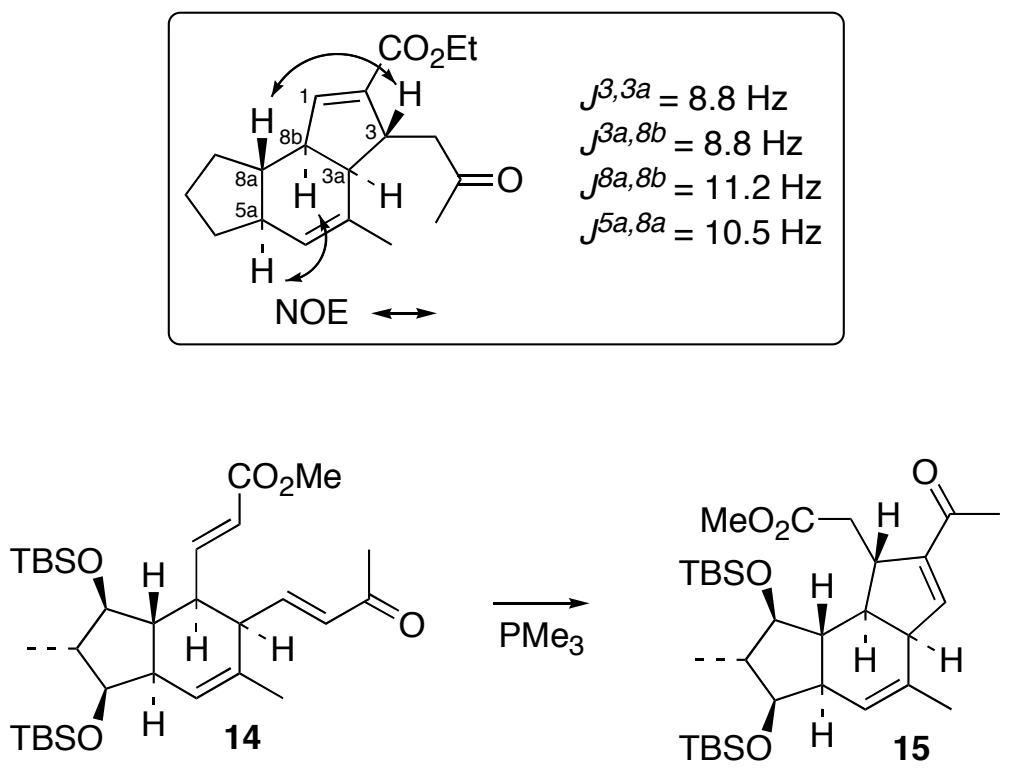

$[1(S), 3 a(S), 5 a(R), 6(R), 7(S), 8(S), 8 \mathrm{a}(R), 8 \mathrm{~b}(S)]$-[2-Acetyl-6,8-bis-(tert-butyldimethylsilanyloxy)-4,7-dimethyl-1,3a,5a,6,7,8,8a,8b-octahydro-as-indacen-1-yl]-Acetic Acid Methyl Ester. A solution of the enone $14(50 \mathrm{mg}, 0.089 \mathrm{mmol})$ in THF $(3 \mathrm{~mL})$ was degassed by the freezepump-thaw method. To this solution, deionized water $(1 \mathrm{~mL}$, degassed by freeze-pump-thaw method) was then added followed by trimethylphosphine (35 $\square \mathrm{L}, 0.34 \mathrm{mmol}$ ). The reaction mixture was stirred for $12 \mathrm{~h}$, partitioned between EtOAc and brine, the organic layer was dried $\left(\mathrm{Na}_{2} \mathrm{SO}_{4}\right)$, filtered and concentrated. Chromatography of the crude product on $\mathrm{SiO}_{2}(1: 4$ to $1: 2$ ether/hexanes) gave $42 \mathrm{mg}(84 \%)$ of a $6: 1$ diastereomeric mixture of air-sensitive cycloadducts 15:16, which was then separated by HPLC (21 mm column using 15\% EtOAc/hexanes with a flow rate of $10 \mathrm{~mL} / \mathrm{min}, t_{R}($ major $)=17 \mathrm{~min} ; t_{R}($ minor $\left.)=21 \mathrm{~min}\right)$. 
Data for major diastereomer 15: $R_{f} 0.48$ (EtOAc/hexanes, 1:4); [ []$^{23}{ }_{\mathrm{D}}-100^{\circ}\left(c 1.18, \mathrm{CH}_{2} \mathrm{Cl}_{2}\right)$; ${ }^{1} \mathrm{H}$ NMR (500 MHz) $\square 6.71$ (d, H-3, $J=2.0 \mathrm{~Hz}$ ), 5.53 (br s, H-5), 3.76 (ddd, H-1, $J=9.3,5.1,2.2$ $\mathrm{Hz}), 3.66\left(\mathrm{~s}, \mathrm{CO}_{2} M e\right), 3.62(\mathrm{~d}, \mathrm{H}-6, J=3.4 \mathrm{~Hz}), 3.55(\mathrm{dd}, \mathrm{H}-8, J=7.6,3.4 \mathrm{~Hz}), 3.44$ (br d, H-3a, $J=6.1 \mathrm{~Hz}$ ), 2.59 (dd, H- $\square, J=14.6,4.6 \mathrm{~Hz}), 2.39$ (dd, H- $, J=14.6,9.0 \mathrm{~Hz}$ ), 2.32 (s, COMe), 2.29 (dd, H-8b, $J=11.0,6.6 \mathrm{~Hz}$ ), 1.94 (dt, H-8a, $J=11.0,11.0,7.5 \mathrm{~Hz}), 1.91$ (m, H-5a), 1.81 (dtd, H-7, $J=7.6,7.6,7.6,3.4 \mathrm{~Hz}$ ), 1.81 (br s, Me-4), 1.03 (d, Me-7, $J=7.8 \mathrm{~Hz}$ ), 0.87 (s, $9 \mathrm{H}$ ), 0.85 (s, 9 H), 0.14 (s, 3 H), 0.08 (s, 3 H), 0.03 (2s, 6 H); ${ }^{13} \mathrm{C}$ NMR (100 MHz) $\square$ 196.4, 173.3, 145.2, 145.0, 133.1, 125.5, 84.7, 79.1, 53.2, 52.3, 51.5, 49.3, 47.6, 44.2, 42.5, 36.9, 26.9, 26.1, 25.9, 22.0, 19.3, 18.3, 18.0, -3.3, -4.2, -4.5, -4.6; IR (neat) 2956, 2928, 2856, 1740, 1670, 1462, $1434 \mathrm{~cm}^{-1}$; HRMS (EI), calcd for $\mathrm{C}_{31} \mathrm{H}_{54} \mathrm{O}_{5} \mathrm{Si}_{2} \mathrm{Na} 585.3408[\mathrm{M}+\mathrm{Na}]^{+}$, found $585.3411 \mathrm{~m} / \mathrm{z}$.

Data for minor diastereomer 16: $R_{f} 0.35$ (EtOAc/hexanes, 1:4); [0] ${ }^{23}{ }_{\mathrm{D}}+22^{\circ}\left(c 0.16, \mathrm{CH}_{2} \mathrm{Cl}_{2}\right)$; ${ }^{1} \mathrm{H}$ NMR (500 MHz) $\square 6.82(\mathrm{~d}, \mathrm{H}-3, J=2.9 \mathrm{~Hz}$ ), 5.68 (br s, H-5), 3.73 (d, H-6, J = 4.2 Hz), 3.55 (s, $\mathrm{CO}_{2} \mathrm{Me}$ ), 3.51 (dt, $\mathrm{H}-1, J=8.3,8.3,4.9 \mathrm{~Hz}$ ), 3.42 (dd, H-8, J = 8.5, 4.4 Hz), 3.28 (br d, H-3a, $J=10.5 \mathrm{~Hz}$ ), 2.79 (dd, H- $\square, J=13.2,5.1 \mathrm{~Hz}), 2.68(\mathrm{q}, \mathrm{H}-8 \mathrm{~b}, J=9.5 \mathrm{~Hz}), 2.35(\mathrm{~s}, \mathrm{COMe}), 2.17$ (dd, H-马, $J=13.3,8.6 \mathrm{~Hz}$ ), 2.17 (dt, H-8a, $J=9.7,9.7,5.8 \mathrm{~Hz}$ ), 1.92 (m, H-5a), 1.86 (dtd, H-7, $J$ = 7.3, 7.3, 7.3, 4.6 Hz), 1.76 (br d, Me-4, $J=1.7 \mathrm{~Hz}), 1.07$ (d, Me-7, $J=7.6 \mathrm{~Hz}), 0.92(\mathrm{~s}, 9 \mathrm{H})$, 0.90 (s, 9 H), 0.12 (s, 3 H), 0.08 (3s, 9 H); ${ }^{13} \mathrm{C}$ NMR (125 MHz) $\square$ 196.9, 173.6, 149.0, 143.7, 135.9, 124.2, 86.8, 78.5, 53.4, 51.5, 50.7, 47.0, 45.8, 44.3, 42.4, 37.5, 27.1, 26.2, 26.1, 26.0, 20.8, 18.9, 18.3, 18.1, -3.6, -4.0; IR 2955, 2930, 2886, 2857, 1738, 1674, 1472, 1463, $1434 \mathrm{~cm}^{-1}$; HRMS (EI), calcd for $\mathrm{C}_{31} \mathrm{H}_{54} \mathrm{O}_{5} \mathrm{Si}_{2} \mathrm{Na} 585.3408[\mathrm{M}+\mathrm{Na}]^{+}$, found $585.3399 \mathrm{~m} / \mathrm{z}$.

Stereochemical assignments for $\mathbf{1 5}$ and $\mathbf{1 6}$ are based on ${ }^{1} \mathrm{H}$ NMR coupling contants and NOE data:

$\begin{gathered}\text { major (desired) } \\ \text { diastereomer }\end{gathered}$
$j^{3 a, 8 b}=6.6 \mathrm{~Hz}$
$J^{8 a, 8 b}=11.0 \mathrm{~Hz}$



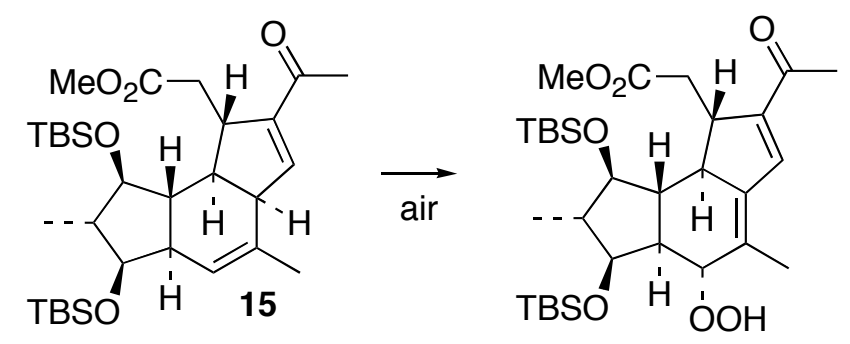

\section{$[1(S), 5(R), 5 a(S), 6(S), 7(R), 8(S), 8 \mathrm{a}(R), 8 \mathrm{~b}(R)]$-[2-Acetyl-6,8-bis-(tert-butyldimethyl-silan-} yloxy)-5-hydroperoxy-4,7-dimethyl-1,5,5a,6,7,8,8a,8b-octahydro-as-indacen-1-yl]-acetic

Acid Methyl Ester. The Morita-Baylis-Hillman product $15(5 \mathrm{mg})$ was allowed to stand neat exposed to the atmosphere for three days before being chromatographed on $\mathrm{SiO}_{2}$ (EtOAc/hexanes, 1:4 to $1: 1)$. This gave $3.8 \mathrm{mg}(72 \%)$ of the oxidation product as a single diastereomer: $R_{f} 0.15$ (EtOAc/hexanes, 1:4); ${ }^{1} \mathrm{H}$ NMR $(500 \mathrm{MHz}) \square 7.31$ (br, OOH), 7.12 (d, H$3, J=2.0 \mathrm{~Hz}), 4.71(\mathrm{~d}, \mathrm{H}-5, J=8.3 \mathrm{~Hz}), 3.86(\mathrm{~d}, \mathrm{H}-6, J=3.4 \mathrm{~Hz}), 3.67(\mathrm{dd}, \mathrm{H}-8, J=7.3,3.4$ $\mathrm{Hz}$ ), 3.60 (s, $\mathrm{CO}_{2} \mathrm{Me}$ ), 3.30 (dd, $\mathrm{CHCO}_{2} \mathrm{Me}, J=15.6,4.6 \mathrm{~Hz}$ ), 3.19 (br d, H-1, J= 3.4 Hz), 2.63 (dd, $\mathrm{CHCO}_{2} \mathrm{Me}, J=15.6,3.7 \mathrm{~Hz}$ ), 2.49 (m, H-8b), 2.39 (s, COMe), 2.17 (ddd, H-8a, J = 13.4, 10.5, 7.3 Hz), 2.11 (ddd, H-5a, $J=13.4,8.3,3.4 \mathrm{~Hz}$ ), 1.97 (dq, H-7, $J=7.8,7.8,7.8,3.4 \mathrm{~Hz}$ ), 1.92 (br d, Me-4, $J=1.0 \mathrm{~Hz}), 1.11$ (d, Me-7, $J=7.6 \mathrm{~Hz}), 0.88$ (2s, $18 \mathrm{H}), 0.13$ (s, $3 \mathrm{H}), 0.12$ (s, 3 $\mathrm{H}), 0.08$ (s, $3 \mathrm{H}), 0.06$ (s, $3 \mathrm{H}) ;{ }^{13} \mathrm{C}$ NMR (100 MHz) $\square$ 197.6, 173.1, 149.4, 145.3, 138.2, 130.1, 84.9, 84.7, 52.4, 51.6, 51.4, 50.5, 45.9, 44.5, 35.0, 27.4, 26.0, 19.3, 18.2, 18.1, 16.0, -2.7, -4.2, 4.7, -4.8; IR (neat) 3392, 2955, 2930, 2897, 2857, 1736, 1666, 1638, $1571 \mathrm{~cm}^{-1}$; HRMS (EI), calcd for $\mathrm{C}_{31} \mathrm{H}_{54} \mathrm{O}_{7} \mathrm{Si}_{2} \mathrm{Na} 617.3306[\mathrm{M}+\mathrm{Na}]^{+}$, found 617.3311 .

The ${ }^{1} \mathrm{H}$ NMR resonance at $7.31 \mathrm{ppm}$ disappears in $\mathrm{D}_{2} \mathrm{O} / \mathrm{CDCl}_{3}$.

Stereochemical assignments for this oxidation product are based on ${ }^{1} \mathrm{H}$ NMR coupling contants and NOE data:

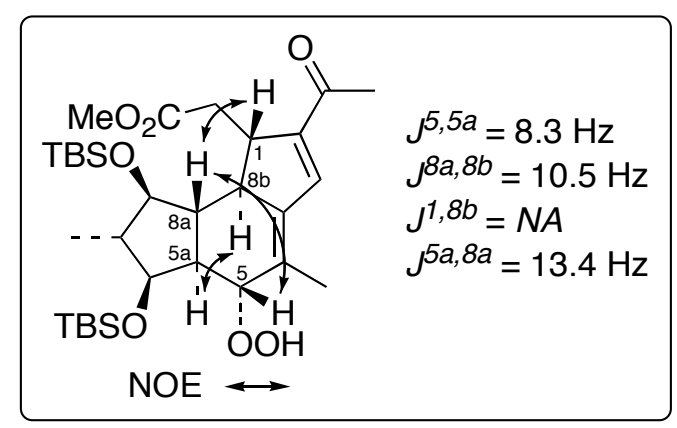

